\title{
Functional CTLA-4 variants associate to both allergic asthma and rhinitis potentially by modulating naïve regulatory $\mathrm{T}$ cells
}

\author{
Anand Kumar Andiappan ${ }^{1}$, Kia Joo PUAN ${ }^{1}$, Yang Yie Sio ${ }^{2}$, Fauziah ALLY ${ }^{1}$, Bernett \\ $\mathrm{LEE}^{1}$, Sri Anusha MATTA ${ }^{2}$, Nurhashikin YUSOF${ }^{1}$, Anis LARBI ${ }^{1}$, De Yun Wang ${ }^{3}$, Fook \\ Chew $^{2}$, and Olaf Rotzschke ${ }^{1}$ \\ ${ }^{1}$ Singapore Immunology Network \\ ${ }^{2}$ National University of Singapore Department of Biological Sciences \\ ${ }^{3}$ National University of Singapore
}

March 5, 2022

Article type: Letter to the Editor

Functional $C T L A-4$ variants associate to both allergic asthma and rhinitis potentially by modulating naïve regulatory $T$ cells

To the editor,

A genome-wide linkage study previously indicated the chromosome $2 \mathrm{q}$ region containing the cytotoxic Tlymphocyte protein $4(C T L A-4)$ gene might be a candidate asthma locus (1). However, prior reports of associations between $C T L A-4$ genetic variants and asthma are conflicting and inconclusive (2-4). This study aimed to determine the role of $C T L A-4$ single nucleotide polymorphisms (SNPs) on allergy risk by carrying out genetic association and functional analyses.

We identified 5 tag-SNPs (rs733618, rs4553808, rs16840252, rs231775, and rs3087243) in CTLA-4 from the Hapmap Chinese Han population (CHB, Figure S1). These tag-SNPs were genotyped in a cohort of 1703 Singapore Chinese adults (age: $22.2 \pm 5.6,42 \%$ male, table S1). Of these 5CTLA-4 tag-SNPs genotyped, rs3087243 has the highest significance level of associations with AR without asthma $\left(p=4.69 \times 10^{-3}\right.$, OR = $1.38)$, asthma without $\mathrm{AR}\left(p=3.49 \times 10^{-4}, \mathrm{OR}=1.58\right)$, and AR with asthma $\left(p=6.67 \times 10^{-4}, \mathrm{OR}=1.52\right.$, Table 1 ).

Next, we determined if rs3087243 has a functional effect onCTLA-4. We extracted CTLA-4 mRNA expression data from 31,300 whole blood samples constituting 36 different cohorts collected by the eQTLgen consortium. In these cohorts, meta-analysis showed a strong correlation between allele "A" of rs3087243 and increasing $C T L A-4$ mRNA expression (meta $p$-value $=2.67 \mathrm{E}-69$, combined $Z$-score $=17.60$, Figure 1A ). From the HapMap CHB data, tag-SNP rs3087243 is in a strong linkage disequilibrium $\left(\mathrm{r}^{2}=1\right)$ with rs11571316 that is located $\sim 1.4 \mathrm{~kb}$ upstream of the CTLA-4 gene (Figure S1 ). We cloned $-1686 \mathrm{bp}$ to +110 bp of the $C T L A$-4gene to conduct an in vitro luciferase assay and showed a significantly higher $C T L A-4$ promoter activity associated with allele "A", as compared to allele "G" of rs3087243 ( $p<0.01$, Figure 1B and 1C ). This suggests rs11571316 might represent a functional SNP that influences the CTLA-4promoter activity and causes the apparent genetic association between the tag-SNP rs3087243 and allergy.

Lastly, when analysing the CTLA-4 surface expression of T cells by flow cytometry we demonstrated a functional effect of rs11571316 on protein levels in T regulatory (Treg) cells. On peripheral blood mononuclear cell (PBMC) samples from a Singapore Chinese cohort $(n=201$, age: $21.0 \pm 2.3,58 \%$ male) we observed robust CTLA-4 expression on Treg cells (gated for CD4+ CD25+ Foxp3+), while only lower amounts of the 
protein were detected on CD25-Foxp3- T effector (Teff) cells (Figure S2 ). Compared to memory-type Treg (CD45RA-) the basal levels in naïve-type Treg cells (CD45RA+) were also lower. However, in this subset we observed a dosage effect of increasing CTLA-4 protein levels from the "CC" to "TT" genotype of rs11571316 (ANOVA $p=8.5 \times 10^{-10}$,Figure 1D ). Besides this strong effect on naïve Treg, nominal association between rs11571316 genotype and CTLA-4 protein levels was only observed in T effector (Teff) cells (ANOVA $p$ $=0.011$,Figure S3 ). This suggests that rs11571316 might regulate $C T L A-4$ protein levels mainly in naïve Treg cells and contribute to the development of allergy. In agreement with this, prior reports have also demonstrated an important role of Treg cells in the control of airway inflammation in asthmatic patients $(5$ ). Also, a separate linkage block in $2 \mathrm{q}$ was previously reported to modulate the mRNA expression of ICOS among Treg and Teff subsets and associated with the risk of atopy and asthma $(6)$. Therefore, we speculated an interplay between ICOS and CTLA-4 in these T cell subsets might contribute to the pro-inflammatory condition that underlies allergy manifestation (Figure S4 ).

In conclusion, the present study identified a functional genetic signal that was associated with allergy via modulating CTLA-4 expression in Treg cell subsets. Our data highlight a possible mechanism for how a targeted immune dysregulation can affect the risk for multiple disease phenotypes. The naïve Treg phenotype warrants further investigation as a potential target for therapeutic intervention in affected patients by harnessing strategies to attenuate CTLA-4 expression on these cells.

\section{References}

1. M. Wjst et al. , A genome-wide search for linkage to asthma. German Asthma Genetics Group. Genomics $\mathbf{5 8}, 1-8(1999)$.

2. M. C. Munthe-Kaas et al., CTLA-4 polymorphisms in allergy and asthma and the TH1/ TH2 paradigm. J Allergy Clin Immunol114, 280-287 (2004).

3. T. D. Howard et al. , Fine mapping of an IgE-controlling gene on chromosome 2q: Analysis of CTLA4 and CD28. The Journal of allergy and clinical immunology 110, 743-751 (2002).

4. N. Hizawa et al. , Increased total serum IgE levels in patients with asthma and promoter polymorphisms at CTLA4 and FCER1B. J Allergy Clin Immunol 108, 74-79 (2001).

5. K. Jansen et al. , T regulatory cells from atopic asthmatic individuals show a Th2-like phenotype. Allergy , (2021).

6. A. K. Andiappan et al., Genetic variants of inducible costimulator are associated with allergic asthma susceptibility. J Allergy Clin Immunol 135, 556-558 (2015).

Author names: Anand Kumar ANDIAPPAN ${ }^{1} \mathrm{PhD}$, Kia Joo PUAN ${ }^{1} \mathrm{PhD}$, Yang Yie $\mathrm{SIO}^{2} \mathrm{PhD}$, Fauziah ALLY $^{1}$, Bernett LEE ${ }^{1} \mathrm{PhD}$, Sri Anusha MATTA ${ }^{2} \mathrm{PhD}$, Nurhashikin YUSOF ${ }^{1}$, Anis LARBI ${ }^{1} \mathrm{PhD}^{2}$, De Yun WANG $^{3}$ MD PhD, Fook Tim $\mathrm{CHEW}^{2 *} \mathrm{PhD}$, Olaf ROTZSCHKE ${ }^{1, *}$ PhDAffiliations: ${ }^{1}$ Singapore Immunology Network (SIgN), \#04-06, 8A Biomedical Grove, Singapore.

2 Department of Biological Sciences, National University of Singapore, Singapore

${ }^{3}$ Department of Otolaryngology, National University of Singapore, Singapore

*Correspondence : Olaf ROTZSCHKE : Olaf_Rotzschke@immunol.a-star.edu.sg; or Fook Tim CHEW : dbscft@nus.edu.sg

Acknowledgements: The authors would like to thank the volunteers for participating in this study, Dr. Ramani Anantharaman and Parate Pallavi Nilkanth for help with sample collection.

Funding: Olaf ROTZSCHKE and Fook Tim CHEW have received research support from the Singapore Ministry of Education Academic Research Fund, Singapore Immunology Network (SIgN), National Medical Research Council (NMRC) (Singapore), Biomedical Research Council (BMRC) (Singapore), 
and the Agency for Science Technology and Research (A*STAR) (Singapore); Grant Numbers: N-154000-038-001, R-154-000-191-112, R-154-000-404-112, R-154-000-553-112, R-154-000-565-112, R-154-000-630112, R-154-000-A08-592, R-154-000-A27-597, R-154-000-A91-592, R-154-000-A95-592, R-154-000-B99-114, BMRC/01/1/21/18/077, BMRC/04/1/21/19/315, BMRC/APG2013/108, SIgN-06-006, SIgN-08-020, NMRC/1150/2008, and H17/01/a0/008. Fook Tim CHEW has received consulting fees from Sime Darby Technology Centre; First Resources Ltd; Genting Plantation, and Olam International, outside the submitted work. Data generated by the SIgN Immunomonitoring platform was supported by a BMRC IAF 311006 grant and BMRC transition funds \#H16/99/b0/011. Dr. Anand Kumar Andiappan was supported by NMRC YIRG grant "OFYIRG17nov065" and A*STAR CDA (Career Development Award) grant (202D800012) and fellowships from the EAACI (European Academy of Allergy and Clinical Immunology) and ERS (European Respiratory Society). Y.Y.S. has received research support from the NUS Resilience \& Growth Postdoctoral Fellowships with grant number: R-141-000-036-281. All funding agencies had no role in the study design, data collection and analysis, decision to publish, or preparation of the manuscript.

Table 1: Genetic association of CTLA-4 tag SNPs to allergic rhinitis and asthma in Singapore Chinese population

Logistic regression test was performed adjusting for age and gender. CI: Confidence Interval; Logistic P: adjusted logistic regression p-value; OR: adjusted odds ratio, using minor allele as the reference category. ${ }^{*}$ Logistic $\mathrm{P}<0.05$ is considered significant association

\begin{tabular}{lll}
\hline Reference/Phenotype & Reference/Phenotype & $\begin{array}{l}\text { AR without Asthma (N=455) vs Non-atopic Non-allergic c } \\
\text { SNP }\end{array}$ \\
Allele & Logistic P \\
rs733618 & G/A & $\mathbf{3 . 0 4 E - 0 3 *}$ \\
rs4553808 & T/C & $9.08 \mathrm{E}-01$ \\
rs16840252 & A/G & $7.96 \mathrm{E}-01$ \\
rs231775 & A/G & $\mathbf{9 . 2 4 E - 0 3 *}$ \\
rs3087243 & 4.69E-03* \\
\hline
\end{tabular}

\section{Figure Legend}

Figure 1. Functional association between 2q variants and $\boldsymbol{C T L A}-4$ expression. (A) Meta-analysis of whole blood eQTL association between CTLA-4 mRNA expression and rs3087243 genotypes, using 31,300 samples constituting 36 different cohorts from the eQTLgen consortium. (B) Schematic illustration of the CTLA-4 gene region with the relative location of SNPs. The cloned region and haplotypes used for the in vitro luciferase assay were also indicated. (C) Relative luciferase units (RLUs) of the CTLA-4 promoter construct were compared across haplotypes. Promoter-less luciferase plasmid pGL4.10 (empty vector, EV) was used as a background control. Lipo: Lipofectamine transfection reagent.(D) Protein expression of CTLA-4 in naïve-type CD45RA + T regulatory (Treg) cells was compared across rs11571316 genotypes. An independent t-test was conducted to determine statistical significance in (C) and (D) that was defined as $p$ -value $<0.05$. 

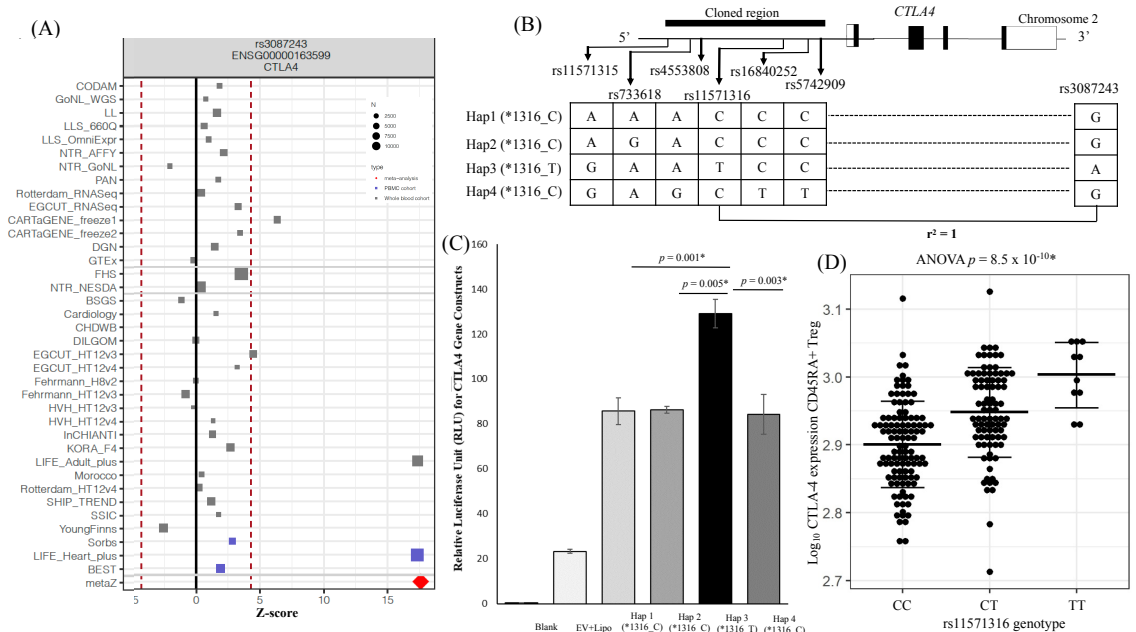